\title{
L'induction neurale chez la souris
}

\section{Mise en place}

L'induction neurale est le premier signe de développement du système nerveux chez les vertébrés, mais c'est aussi une des questions les plus mal connues de l'embryogenèse précoce. Chez les amphibiens, depuis la fameuse définition par Spemann du centre organisateur [1], on admet que l'induction neurale doit avoir lieu pendant la gastrulation: l'ectoderme est induit à former la plaque neurale par un signal issu du mésoderme dorsal sous-jacent (le centre organisateur; pour revue, voir [2]). De très nombreux travaux chez le xénope ont permis d'identifier plusieurs molécules de signalisation cellulaire impliquées dans le processus d'induction neurale (pour revue, voir [3]). Certaines répondent même aux critères de définition d' «inducteur neural ", c'est-à-dire pouvant induire la formation de neuroectoderme en l'absence de mésoderme. Ces molécules semblent devoir agir en combinaison afin de spécifier, par exemple, l'expression de marqueurs antérieurs et postérieurs. En effet, dans un essai in vitro, Noggin seule induit préférentiellement l'expression de marqueurs antérieurs [4] alors que l'ajout de bFGF permet d'observer l'expression de marqueurs postérieurs [5]. Un modèle d'induction neurale en deux étapes a donc été proposé, impliquant une première étape lors de laquelle l'ectoderme serait «commis» d'abord à un destin neural antérieur puis, à mesure que le mésoderme progresse, celui-ci induirait une postériorisation progressive [6, 7]. L'induction neurale, comme de nombreux autres processus du développement, ne répond probablement pas à la formule «une seule cause un seul effet", d'autant que la redondance d'informations semble être la règle dans les étapes cruciales du développement des vertébrés.

Chez l'embryon de souris aussi, l'induction neurale est une caractéristique de la gastrulation. La gastrulation débute à 6,5 jours de gestation, par l'induction du mésoderme et la formation de la ligne primitive, marquant l'extrémité postérieure de l'embryon (figure 1b). L'axe antéropostérieur est ainsi établi. La ligne primitive va progresser jusqu'à l'extrémité distale de l'embryon; le mésoderme s'intercale entre l'endoderme et l'ectoderme. Au cours de sa progression, à l'extrémité antérieure de la ligne primitive, va se constituer le centre organisateur ou nœud (node) par analogie avec le noud de Hensen chez le poulet, bientôt luimême précédé par un groupe de cellules mésodermiques constituant la plaque préchordale (figure 1d). Le nœud est à l'origine de l'endoderme définitif et de la notochorde, source de signaux inducteurs impliqués notamment dans la régionalisation du tube neural [9]. Enfin, vers 7,5 jours de développement, la plaque neurale se forme comme un épaississement de l'ectoderme, en avant de la ligne primitive, et s'étend par addition de cellules neuroectodermales nouvellement recrutées à son extrémité postérieure. Selon des mécanismes similaires à ceux œuvrant chez les vertébrés inférieurs (pour revue, voir [10]) l'épiblaste a été induit à former du neuroectoderme par des signaux émanant du mésoderme axial sous-jacent (préchordal et notochordal émergeant de l'extrémité rostrale de la ligne primitive). Le neuroectoderme ainsi formé montre très rapidement des signes de régionalisation par l'expression localisée de différents marqueurs, dont certains sont déjà même exprimés dans l'épiblaste avant la gastrulation, tels Otx2 ou Sox2 (J. Collignon et R. Lowell-Badge, communication personnelle et [11]).

\section{Détermination précoce}

Chez le minuscule embryon de souris, l'induction du développement neural est donc intimement associée dans le temps et dans l'espace à l'induction et la régionalisation du mésoderme. Peut-on découpler ces deux événements? L'épiblaste acquiert-il un stade de compétence avant cette induction, est-il même prérégionalisé? Les signaux reçus du mésoderme sous-jacent sont-ils de type vertical ou «planaire» (pour revue, voir [12])? Les approches de l'embryologie expérimentale chez la souris ont connu d'énormes progrès: il est possible de garder des embryons, prélevés après l'implantation, en culture in vitro, pendant plus de 36 heures ainsi que des explants de feuillets primitifs. Les expériences de lignage cellulaire et de greffes sont réalisables. Les cartes de territoires présomptifs établies par différents groupes, selon différentes techniques, marquage de cellules, individuellement ou par petits groupes, greffes homo ou hétérotopiques sont relativement homogènes [13-16]. Ces travaux permettent de conclure que la région destinée à devenir le neuroectoderme se situe à l'extrémité distale de l'embryon, ce qui en fait donc le tissu cible pour l'induction 


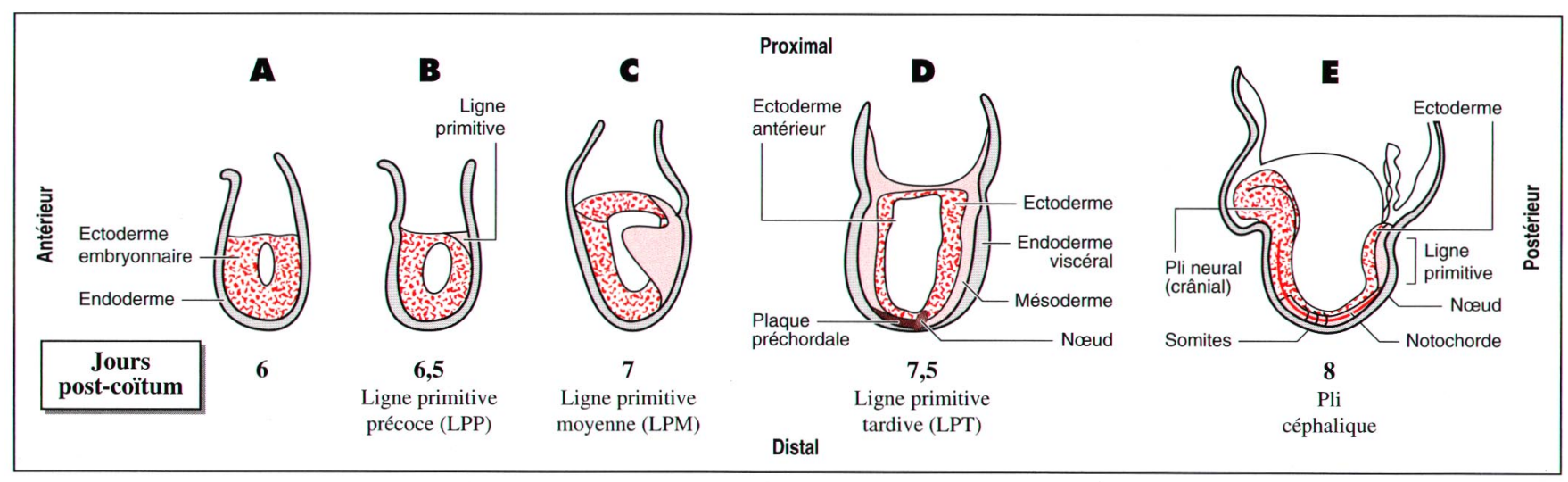

Figure 1. Représentation schématique des différentes étapes de la gastrulation chez la souris. L'embryon en gastrulation a en 3 dimensions une forme de tasse, ou de doigt de gant, enfermant la cavité proamniotique en son centre. Les annexes embryonnaires ne sont pas figurées. A. Au stade dit "œuf cylindre", l'embryon est constitué de deux feuillets embryonnaires l'ectoderme primitif, appelé aussi épiblaste et l'endoderme; B. formation de la ligne primitive à l'extrémité postérieure, le mésoderme s'intercale entre les deux feuillets; $\mathbf{C}$. stade de la ligne primitive moyenne, le mésoderme progresse vers l'extrémité distale et aussi vers l'avant de l'embryon; D. formation du nœud et de la plaque préchordale; E. stade dit du pli céphalique, avec apparition des différents tissus; ne sont schématisés que ceux qui sont utiles à la discussion. Ectoderme: en pointillé rouge, endoderme: en gris clair, mésoderme: en rose; nœud, plaque préchordale, notochorde: en rouge.

neurale [16]. L'ectoderme antérieur est déjà déterminé à devenir du neuroectoderme dès le stade de ligne primitive tardive [13].

\section{Un centre organisateur chez les mammifères}

Une spectaculaire et concluante expérience de greffe a récemment permis de démontrer que le nœud de l'embryon de souris a des propriétés inductrices tout à fait voisines de celles du centre organisateur du xénope [17]. La greffe sur un embryon hôte, en région postéro-latérale, du nœud prélevé chez un embryon donneur de même stade, ligne primitive tardive, induit la formation d'un second axe neural. C'est-à-dire une notochorde ectopique, tandis qu'un pli neural et des somites ectopiques sont formés en recrutant les cellules de l'hôte. Le nœud chez la souris fonctionne donc comme un centre organisateur et serait aussi responsable de l'émission des signaux "verticaux " dans l'induction neurale. Mais le nœud est aussi une structure complexe dont les différents territoires n'ont pas tous les mêmes capacités inductrices, comme cela a été montré chez le poulet [18]; les mêmes expériences paraissent impossibles à faire chez la souris.

\section{Inductions : régionalisations} des signaux

D'élégantes expériences in vitro de combinaison d'explants de feuillets embryonnaires prélevés à différents stades de la gastrulation $[19,20]$ apportent des précisions intéressantes sur le déroulement et les protagonistes de l'induction neurale. Mis en contact avec des explants d'ectoderme, le mésendoderme* a des capacités d'induction différentes selon sa provenance sur l'axe antéro-postérieur et le stade de la gastrulation, ce qui a aussi été observé chez le xénope. L'ectoderme antérieur prélevé au moment de la mise en place de la ligne primitive $(6,5 \mathrm{jpc})$, avant tout

\footnotetext{
* Le terme de mésendoderme est proposé par les auteurs pour préciser que l'explant de mésoderme peut contenir des cellules endodermiques, notamment de la notochorde [19].
}

contact direct avec du mésoderme, est capable de répondre par l'expression de marqueurs de différenciation neurale quand il est mis en présence de ces signaux inducteurs. L'ectoderme antérieur isolé en culture à un stade légèrement plus avancé de la gastrulation (ligne primitive moyenne, 7 jpc) est déjà spécifié, de façon autonome par rapport aux cellules environnantes (cell autonomous), à exprimer le gène En2, normalement exprimé plus de 12 heures plus tard dans le cerveau postérieur in vivo. Les capacités de réponse de l'ectoderme varient également selon son stade de développement et sa provenance, l'ectoderme postérieur au stade «pli céphalique» (8 jpc, figure 1e) est encore compétent à répondre aux signaux émis par le mésendoderme. Ces deux observations montrent que l'ectoderme présenterait, d'une part, un stade de compétence préliminaire (ou bien serait-ce la première manifestation de l'induction neurale?), et, d'autre part, une spécification installée très précocement, sans contact préalable direct avec le mésendoderme et/ou le mésoderme préchordal, mais peutêtre relayée dans l'ectoderme. Cela 
suggère une possible propagation des signaux inducteurs de type horizontal, dans le même plan, dont la démonstration définitive, et de l'existence et de l'origine, nécessiterait des expériences encore plus fines.

Le gène Otx2 est exprimé dans tout l'épiblaste avant gastrulation mais, dès la fin de la gastrulation, son expression dans le neuroectoderme est restreinte à un domaine antérieur correspondant au futur système nerveux central, chez l'embryon de souris aussi bien que de poulet ou de xénope [11]. Les expériences de recombinaison d'explants mettent en évidence un effet répresseur du mésendoderme postérieur et un effet positif, activateur, issu du mésendoderme antérieur dans la respécification de l'expression d'Otx2 dans l'ectoderme antérieur [21]. Ces spécificités régionales et l'intervention de régulations positives et négatives indiquent la multiplicité probable des signaux et rappellent le modèle d'induction neurale en deux étapes proposé chez les amphibiens.

\section{Molécules inductrices : les candidats possibles}

Les précisions ainsi apportées sur la fenêtre de temps et les tissus protagonistes de l'induction neurale chez la souris présentent une ressemblance certaine avec l'orchestration générale de ces processus chez les vertébrés inférieurs. Il reste à identifier les molécules responsables de la transmission de ces signaux inducteurs. On connaît plusieurs molécules de signalisation cellulaire ainsi que des facteurs de transcription exprimés dans les régions concernées, ligne primitive, nœud, plaque préchordale, au cours de la gastrulation (pour revue, voir [3, 9, 22, 23]). Pour la plupart d'entre eux, leurs gènes ont été clonés sur la base de la conservation d'analogie avec des gènes identifiés chez d'autres organismes et impliqués dans ces processus d'induction. Plusieurs facteurs de croissance de type FGF, et leurs récepteurs sont exprimés avant et au cours de la gastrulation, dans l'ectoderme ou bien le mésoderme de la ligne primitive (pour revue, voir [23]). L'expression de goosecoid, nodal, HNF3 $\beta$, T et Lim-1, caractérise le nœud; $T$ et $H N F-3 \beta$ sont aussi exprimés dans la notochorde, T et Lim-1 dans la ligne primitive [24]. Nous allons examiner ce que peuvent nous apprendre les phénotypes des mutants de perte de fonction de certaines de ces molécules sur les mécanismes de l'induction neurale, tout en considérant ses liens étroits avec la régionalisation du mésoderme (voir Tableau I). Les mutations nulles présentant un phénotype létal entre l'implantation et le début de la gastrulation témoignent certainement du rôle crucial du gène affecté mais restent peu informatives pour l'analyse de l'induction neurale. Parmi cette catégorie, on trouvera $\mathrm{Fg} f$, Evx-1, $\beta 1$-integrin, etc. (pour revue, voir [25]). Il faudra attendre des outils de mutation conditionnelle pour élucider leur rôle. De même, les phénotypes tardifs, viables ou létaux audelà de 11-12,5 jpc, nous indiquent, que le gène atteint ne joue pas le rôle attendu d'après son profil d'expression à la gastrulation ou son rôle chez d'autres organismes. Ou bien encore, l'effet de son absence peut être compensé par un gène voisin, connu ou inconnu. Ce groupe inclut notamment les gènes codant pour les facteurs de croissance FGF3 et FGF5 et la protéine goosecoid. Les souris mutantes pour le gène goosecoid, facteur de transcription à homéodomaine, exprimé transitoirement dans le nœud au début de la gastrulation, ne présentent pas un phénotype anormal à la gastrulation, mais naissent à terme avec des malformations de la face, corrélées à la phase d'expression tardive de ce gène [26].

\section{Les partenaires essentiels de l'induction neurale}

Le gène codant pour nodal, une protéine sécrétée de la superfamille des facteurs de croissance de type TGF $\beta$, a été identifié par mutagenèse par insertion de rétrovirus. Les embryons homozygotes nuls pour l'expression de ce gène ne dépassent pas le cap de la gastrulation: le noud et la notochorde ne se forment pas. Quelques cellules de type mésodermique et de l'ectoderme sont détectées, mais il n'y a pas d'expression de goosecoïd [27]. Nodal est donc indispensable à la formation du noud, vraisemblablement liée à la mise en route de la gastrulation, et par conséquent à l'induction neurale.

Les embryons mutants homozygotes pour l'inactivation du gène $H N F 3 \beta$ sont dépourvus de nœud et de notochorde et présentent des anomalies importantes de l'organisation de l'axe dorso-ventral [21, 28]. Une activité de type «organisateur» persiste cependant: quelques cellules expriment goosecoid mais ne s'organisent pas en nœud. Du mésoderme dorsal, et du tissu neural sont formés, avec le maintien de l'expression de plusieurs marqueurs, désorganisée mais respectant l'ordre antéro-postérieur. Néanmoins, la moitié des embryons mutants sont dépourvus de structures antérieures au cerveau postérieur. La variabilité des phénotypes mutants est un phénomène fréquemment observé qui peut s'expliquer par les différences de contexte génétique entre les individus. De même, une variabilité est observée pour une mutation donnée selon la souche de souris dans laquelle elle est introduite.

La mutation nulle du gène codant pour la protéine FGFR-1, récepteur des FGF, provoque chez les embryons homozygotes des défauts de croissance et de la régionalisation du mésoderme axial et postérieur. Certains mutants ne dépassent pas le stade de la ligne primitive: FGFR1 apparaît nécessaire à la progression de la gastrulation [29].

Lim-1 est un gène à homéoboîte exprimé dans le nœud. L'inactivation de ce gène se traduit par l'absence totale de structures céphaliques et de cerveau antérieur jusqu'au niveau des premiers rhombomères; le reste du corps se développe normalement. Les deux marqueurs précoces du nœud, nodal et goosecoid, sont détectés mais «délocalisés» et retardés : la plaque de mésoderme préchordale est absente, un nœud organisé ne se forme que vers 8,5 jpc. Quelques embryons développent parfois même un second axe neural. Lim-1 pourrait bien être un acteur principal d'un effet organisateur partiel [30].

Ce phénotype n'est pas sans rappeler celui des animaux mutants nuls du gène $\operatorname{Otx2}$, qui ont aussi «perdu la tête", dans les mêmes limites, mais présentent plus de défauts d'organi- 
Tableau I

\section{RÉSUMÉ DES CARACTÉRISTIQUES DES GĖNES ÉVENTUELLEMENT} IMPLIQUÉS DANS L'INDUCTION NEURALE

\begin{tabular}{|c|c|c|}
\hline Gène & $\begin{array}{l}\text { Expression normale } \\
\text { à la gastrulation }\end{array}$ & Phénotype homozygote \\
\hline Nodal & nœud & $\begin{array}{l}\text { absence de nœud, létal à la gastru- } \\
\text { lation, à peine formation de méso- } \\
\text { derme }\end{array}$ \\
\hline$H n f-3 \beta$ & nœud, notochorde & $\begin{array}{l}\text { absence de nœud "organisé " et de } \\
\text { notochorde } \\
\text { anomalies de formation de la ligne } \\
\text { primitive } \\
\text { défauts de l'organisation dorso- } \\
\text { ventrale du tube neural } \\
\text { létal embryonnaire }\end{array}$ \\
\hline Lim-1 & nœud, ligne primitive & $\begin{array}{l}\text { absence totale de structures ros- } \\
\text { trales au cerveau postérieur } \\
\text { formation du nœud retardé, létal } \\
\text { embryonnaire }\end{array}$ \\
\hline Otx2 & ectoderme antérieur & $\begin{array}{l}\text { absence de structures rostrales au } \\
\text { cerveau postérieur } \\
\text { absence du nœud et de la plaque } \\
\text { préchordale, anomalie, de l'axe an- } \\
\text { téro-postérieur } \\
\text { létal embryonnaire }\end{array}$ \\
\hline FgfR1 & $\begin{array}{l}\text { ectoderme embryonnaire } \\
\text { mésoderme de la ligne } \\
\text { primitive }\end{array}$ & $\begin{array}{l}\text { létal à la gastrulation }(7,5-9,5 \mathrm{jpc}) \text {; } \\
\text { troubles de croissance et d'exten- } \\
\text { sion de I'axe antéro-postérieur, no- } \\
\text { tochorde et ligne primitive anor- } \\
\text { males }\end{array}$ \\
\hline
\end{tabular}

De nombreux gènes sont exprimés lors de la gastrulation chez la souris, nous nous limitons ici à ceux susceptibles d'être impliqués dans l'induction neurale (dans l'état actuel de nos connaissances).

(nodal: [27]; Hnf-33 : [21, 28]; Lim-1: [30]; Otx2: [31, 32] Ang et al., 1995, communication à Heidelberg; Fgfr1: [22]).

*jpc: jours de développement post-coïtum.

sation de l'axe antéro-postérieur que les embryons Lim1-/- (TableauI); surtout, ils n'ont apparemment plus de nœud ni de plaque préchordale alors que $O t \times 2$ n'y est pas exprimé chez la souris (Ang SL et al., communication au meeting Mouse Molecular Genetics, Heidelberg: 1995 $[31,32])$. Otx2 est un gène à homéoboîte, marqueur précoce du neuroectoderme puis du cerveau antérieur [11].

Quant au gène $B M P-4$, d'après le phénotype létal vers $8,5 \mathrm{jpc}$ des mutants nuls qui présentent des dérivés des trois feuillets embryonnaires atténués par la persistance d'une activité de type "organisateur", puisqu'il y a maintien de l'induction neurale et de la régionalisation antéro-postérieure. Cette activité persistante est corrélée à la présence de cellules exprimant goosecoid, dont l'expression précède de peu celle de $h n f-3 \beta$ dans le développement normal. La disparition des structures antérieures au cerveau postérieur chez certains mutants pourrait s'expliquer par la perte de cette activité. HNF-3 $\beta$ paraît essentiel à la formation de la notochorde, mais n'a probablement pas un rôle de premier plan dans le centre organisateur.

L'observation des embryons Lim1-/- suggère l'existence d'une activité organisatrice de la tête ou des structures antérieures dont ils sont entièrement dépourvus. Cette activité pourrait résider dans la plaque mésodermique préchordale, absente de ces embryons, ou encore résulter de l'absence d'expression appropriée des marqueurs du nœud, dont la formation est retardée d'environ 24 heures (dont le produit du gène $T$ ). Certains de ces embryons présentent un axe neural surnuméraire, comme si l'activité organisatrice du tronc, qui persiste, se trouvait déréprimée et fasse une tentative désespérée d'organisation. Dans ces deux exemples de perte d'organisation des structures antérieures, la limite rostrale des embryons mutants, Lim-1-/- et Otx2-/-, est très similaire et se situe au niveau des premiers rhombomères, ce qui correspond à la limite antérieure des domaines d'expression des gènes Hox. D'autre part, ces régions antérieures du cerveau sont parmi les derniers acquis du système nerveux central des Vertébrés. Spemann avait observé une "dichotomie» fonctionnelle de l'organisateur: le mésoderme dorsal et antérieur induit préférentiellement des axes secondaires constitués de structures antérieures, le mésoderme à devenir postérieur induit plutôt des structures postérieures. C'est peut-être cette activité organisatrice du tronc ou postérieure qui est affectée chez les mutants du gène $T$, qui présentent de sévères malformations de la noto- 
chorde et une troncature des structures postérieures, ou encore chez les embryons Wnt3a-/- dont le développement postérieur dépasse juste les membres antérieurs.

Le phénotype des mutants Otx2-/apporte un corollaire intéressant à ces observations. Otx2 semble être un acteur précoce de la spécification du neuroectoderme. Mais l'étendue de ce phénotype est peut-être corrélée à la phase d'expression d'Otx2 dans l'épiblaste avant la gastrulation. L'absence précoce d'Otx2 dans la cascade de régulation pourrait-elle avoir un effet indirect sur la formation du nœud et de la plaque préchordale par le biais d'un dialogue entre le mésoderme et l'ectoderme ou par absence de cibles pour les signaux réglant l'expression d'Otx2 à la gastrulation? $\mathrm{Ou}$ bien est-on passé à côté de l'expression d'Otx2 dans le nœud? Son homologue chez le xénope est en effet exprimé dans le centre organisateur et aurait une activité inductrice [33].

Les phénotypes observés à la suite de mutations nulles ciblées rendent compte non seulement de l'effet direct de la mutation mais aussi des conséquences des déséquilibres introduits dans le jeu des interactions avec les autres molécules impliquées dans le processus. Et le dernier exemple illustre bien ce point. La notion de signal inducteur doit être considérée comme un ensemble dynamique de signaux.

\section{Conclusion}

Ce survol rapide et encore incomplet - d'autres molécules, comme noggin ou sonic hedgehog, manquent à l'appel - souligne l'importance du nœud et de la plaque préchordale dans la régionalisation du mésoderme, dans les processus d'induction des territoires antérieurs et vient donc à l'appui des informations fournies par les expériences de greffe et de combinaisons d'explants in vitro. Cela va dans le sens de la conservation de l'orchestration générale des mécanismes de la gastrulation parmi les différents vertébrés. Mais l'exemple de goosecoïd, entre autres, nous rappelle que des différences existent bien, et qu'on ne peut se contenter d'extrapoler. C'est pourquoi il est important de poursuivre les approches d'embryologie expérimentale chez la souris et de développer un système permettant de tester un éventuel inducteur neural. De l'examen présent, l'induction neurale apparaît comme un processus instructif adressé à une cible permissive. Des travaux, chez le xénope, ont montré que l'induction neurale pouvait parfois sembler résulter d'une levée d'inhibition (pour revue, voir [12, 34]). L'induction neurale met-elle en œuvre des régulations positives et négatives comme on peut l'observer plus tard dans le développement (voir [35])? L'embryon de souris aborde la gastrulation plus de 6 jours après la fécondation, et ne survit pas sur des réserves issues de l'organisme maternel. Entre-temps l'activation transcriptionnelle a eu lieu, et l'embryon a dû s'implanter et donc développer des annexes embryonnaires et une interface avec le milieu maternel. La liste est longue des molécules synthétisées avant la gastrulation (parfois après aussi) et dont l'inactivation du gène est fatale à l'embryon [25]. La létalité précoce due à la perte de fonction de molécules produites dans l'endoderme viscéral et pariétal révèle l'importance des interactions entre ces tissus et l'embryon pour son développement. Les différences avec des organismes tels que le xénope ou le poulet ne sont donc pas surprenantes. Par exemple, les membres du fameux trio: activine, récepteur de l'activine et follistatine, ont clairement des rôles très différents chez la souris de ce qu'on pouvait attendre à partir des travaux réalisés chez le xénope. Les souris déficientes pour la follistatine ne sont pas du tout dépourvues de système nerveux (pour revue, voir [36]). Le facteur HGF/SF, inducteur neural exprimé dans l'ébauche du nœud de Hensen chez le poulet n'est pas du tout nécessaire chez la souris (pour revue, voir [37]).

Le développement de stratégies d'identification de nouveaux gènes impliqués dans les processus d'induction du mésoderme et de l'induction neurale directement appliquées à la Souris constitue une alternative inévitable pour mieux comprendre ces mécanismes. C'est une approche qui s'est déjà révélée efficace et pourra apporter des éclaircissements sur les probables spécificités de l'embryogenèse murine $[38,39]$

\section{Marie-Odile Ott}

National Institute for Medical Research. The Ridgeway, Mill Hill, London NW7 1AA, Royaume-Uni.

\section{RÉFÉRENCES}

1. Spemann H. Embryonic development and induction. Yale University Press. New Haven, 1938.

2. Condamine H. L'organisateur de l'embryon d'amphibien. médecine/sciences 1982 ; 8: 483-6.

3. Kelly O, Melton DA. Induction and patterning of the Vertebrate nervous system. Trends Genet 1995; 11 : 273-8.

4. Lamb TM, Knecht AK, Smith WC, Stachel SE, Economides AN, Stahl N, Yancopolous GD, Harland RM. Neural induction by the secreted polypeptide noggin. Science 1993; 262: 713-8.

5. Lamb TM, Harland RM. Fibroblast growth factor is a direct neural inducer, which combined with noggin generates anteroposterior neural pattern. Development 1995; 121: 3627-36.

6. Gilbert SF, Saxèn L. Spemann's organizer : model and molecules. Mec Dev 1993; 41: 73-89.

7. Ruiz i Altaba A. Pattern formation in the vertebral neural plate. Trends Neurol Sci $1994 ; 17: 233-43$.

8. Boucaut J, Umbhauer M, Riou J. L'induction du mésoderme. médecine/sciences 1994; $10: 854-67$.

9. Beddington RS, Smith JC. Control of vertebrate gastrulation: inducing signals and responding genes. Curr Op Genet Dev 1993; $3: 655-61$.

10. Joly JS, Cohen-Tannoudji M. L'unité de la gastrulation chez les vertébrés. médecine/sciences $1994 ; 10: 84-90$.

\section{TIRÉS À PART}

M.O. Ott. 


\section{RÉFÉRENCES}

11. Simeone A, Acampora D, Mallamaci A, Stornaiuolo A, D'Apice MR, Nigro V, Boncinelli E. A vertebrate gene-related to orthodenticle contains an homeodomain of the bicoid class and demarcates anterior neuroectoderm in the gastrulating mouse embryon. EMBO J 1993; 12 : 2735-47.

12. Ruiz i Altaba A. Induction and axial patterning of the neural plate: planar and vertical signals. J Neurobiol 1993 ; 24: 1276-304.

13. Beddington RSP. An autoradiographic analysis of tissue potency in different region of the embryonic ectoderm during gastrulation in the mouse. I Embryol Exp Morphol $1982 ; 69:$ 265-85.

14. Tam PPL. Regionalisation of the mouse embryo ectoderm: allocation of prospective ectodermal tissues during gastrulation. Development 1989 ; 107 : 55-67.

15. Lawson KA, Pedersen RA. Clonal analysis of cell fate during gastrulation and early neurulation in the mouse. Postimplantation development in the mouse. Chichester: John Wiley and sons, 1992.

16. Quinlan A, Williams EA, Tan SS, Tam PPL. Neuroectodermal fate of epiblast cells in the distal region of the mouse egg cylinder: implication for body plan organization during early embryogenesis. Development $1995 ; 121: 87-98$.

17. Beddington $\mathrm{R}$. Induction of a second neural axis by the mouse node. Development 1994; 120: 613-20.

18. Storey KG, Selleck MAJ, Stern CD. Neural induction and regionalisation by different subpopulations of cells in Hensen's node. Development 1995; 121: 417-28.

19. Ang SL, Rossant J. Anterior mesendoderm induces mouse Engrailed genes in explant culture. Development 1993 ; 118 : 139-49.

20. Ang SL, Conlon RA, Jim O, Rossant J. Positive and negative signals from mesoderm regulate the expression of mouse Otx2 in ectoderm explants. Development $1994 ; 120: 2979-89$.

21. Ang SL, Rossant J. HNF-3 beta is essential for node and notochord formation in mouse development. Cell 1994; 78: 561-74.

22. Conlon F, Beddington R. Mouse gastrulation from a frog's perspective. Semin Dev Biol 1995; 6 : 249-56.

23. Yamaguchi TP, Rossant J. Fibroblast growth factors in mammalian development. Curr Op Gen Dev 1995; 5 : 485-9.

24. Camus A, Babinet C. Brachyury, un gène essentiel pour la gastrulation et la formation du mésoderme. médecine/sciences 1993; 9: 1118-21.

25. Cross JC, Werb Z, Fisher S. Implantation and the placenta: key pieces of the development puzzle. Science 1994; 266: 1508-17.

26. Yamada G, Mansouri A, Torres M, Stuart ET, Blum M, Schultz M, DeRobertis E, Gruss P. Targeted mutation of the murine goosecoid gene results in craniofacial defects and neonatal death. Development 1995 ; 121: 2917-22.

27. Conlon FL, Lyons KM, Takaesu N, Barth
KS, Kispert A, Herrmann B, Robertson EJ. A primary requirement for nodal in the formation and maintenance of the primitive streak in the mouse. Development 1994; 120 : 1919-28.

28. Weinstein D, Ruiz i Altaba A, Chen WS, Hoodless P, Jessell TM, Darnell JE. The winged-helix transcription factor HNF-3beta is required for notochord development in the mouse embryo. Cell 1994; 78: 575-88.

29. Yamaguchi TP, Harpal K, Henkemeyer $\mathrm{M}$, Rossant J. fgfrl is required for embryonic growth and mesodermal patterning during mouse gastrulation. Genes Dev 1994; 8: 3032-42.

30. Shawlot W, Behringer RR. Requirement for Lim1 in head-organizer function. Nature $1995 ; 374$ : 425-30.

31. Acampora D, Mazan S, Lallemand Y, Avantaggiato V, Maury M, Simeone A, Brûlet $\mathrm{P}$. Forebrain and midbrain regions are deleted in Otx2-/- mutants due to a defective anterior neuroectoderme specification during gastrulation. Development 1995; 121 : 3279-90.

32. Matsuo I, Kuratani S, Kimura C, Takeda $\mathrm{N}$, Aizawa S. Mouse Otx2 functions in the formation and patterning of rostral head. Genes Dev 1995 9: 2646-58.

33. Blitz IL, Cho KWY. Anterior neuroectoderm is progressively induced during gastrulation: the role of the Xenopus homeobox gene orthodenticle. Development 1995; 121: 993-1004.

34. Doniach T. Planar and vertical induction of anteroposterior pattern during the development of the Amphibian nervous system. J Neurobiol 1993; 24 : 1256-75.

35. Simpson P. Positive and negative regulators of neural fate. Neuron 1995; 15 : 739-42.

36. Smith J. Angles on activin's absence. Nature 1995; 374: 311-2.

37. Bronner-Fraser M. Hepatocyte growth factor/scatter factor (HGF/SF) in early development: evidence for a role in neural induction. Trends Genet 1995; 11 : 423-5.

38. Harrison SM, Dunwoodie SL, Arkell $\mathrm{RM}$, Lehrach $\mathrm{H}$, Beddington RSP. Isolation of novel tissue-specific genes from cDNA libraries representing the individual tissue constituents of the gastrulating mouse embryo. Development 1995; 121 : 2479-89.

39. Skarnes WC, Moss JE, Hurtley S, Beddington RSP. Capturing cell surface molecules in mouse embryonic stem cells. Proc Natl Acad Sci USA 1995; 92: 6592-6.

\begin{tabular}{l} 
* GLOSSAIRE * \\
\hline Épiblaste : feuillet embryonnaire d'en- \\
viron 700 cellules avant gastrula- \\
tion et qui est à l'origine de l'en- \\
semble des tissus définitifs de \\
l'embryon et du mésoderme extra- \\
embryonnaire, il est organisé en épi- \\
thélium et est aussi appelé ectoder- \\
me primitif.
\end{tabular}

\section{GLOSSAIRE *}

Ligne primitive : se forme par délamination de cellules de l'épiblaste, qui s'intercalant entre l'ectoderme embryonnaire et l'endoderme viscéral vont constituer le mésoderme; sa formation marque le début de la gastrulation, et l'extrémité postérieure de l'embryon; elle est aussi appelée "sillon primitif».

Noud : structure d'une vingtaine de cellules formée à l'extrémité antérieure de la ligne primitive quand celle-ci atteint la partie distale de l'embryon. C'est une structure bilaminaire car non recouverte par de l'endoderme viscéral.

Mésoderme axial : il serait principalement issu d'une population de cellules du noud et est ainsi nommé par opposition au mésoderme latéral; il est sous-jacent au neuroectoderme du futur cerveau antérieur et moyen.

Plaque préchordale : ensemble de cellules mésodermiques issues par ingression de l'épiblaste au niveau du noud et migrant antérieurement, ainsi appelé car elle "précède » la notochorde. 